\title{
Improving Langley calibrations by reducing diurnal variations of aerosol Ångström parameters
}

\author{
A. Kreuter, S. Wuttke, and M. Blumthaler \\ Division for Biomedical Physics, Department of Physiology and Medical Physics, Innsbruck Medical University, \\ Muellerstr. 44, 6020 Innsbruck, Austria
}

Correspondence to: A. Kreuter (axel.kreuter@i-med.ac.at)

Received: 25 July 2012 - Published in Atmos. Meas. Tech. Discuss.: 7 September 2012

Revised: 18 December 2012 - Accepted: 1 January 2013 - Published: 14 January 2013

\begin{abstract}
Errors in the sun photometer calibration constant lead to artificial diurnal variations, symmetric around solar noon, of the retrieved aerosol optical depth (AOD) and the associated Ångström exponent $\alpha$ and its curvature $\gamma$. We show in simulations that within the uncertainty of state-ofthe-art Langley calibrations, these diurnal variations of $\alpha$ and $\gamma$ can be significant in low AOD conditions, while those of AOD are negligible. We implement a weighted Monte Carlo method of finding an improved calibration constant by minimizing the diurnal variations in $\alpha$ and $\gamma$ and apply the method to sun photometer data of a clear day in Innsbruck, Austria. The results show that our method can be used to improve the calibrations in two of the four wavelength channels by up to a factor of 3.6.
\end{abstract}

\section{Introduction}

Sun photometers (SPM) are radiometers to determine the spectral aerosol optical depth (AOD), the columnar aerosol loading of the atmosphere, which is an important radiative forcing parameter of the Earth's climate system (Forster et al., 2007). The AOD is determined from direct sun irradiance measurements and application of the Bouguer-LambertBeer law which describes the exponential extinction of light on its way through the atmosphere. The main challenges in sun photometry are the calibration as well as ensuring a constant filter transmission (Shaw, 1976).

The best sun photometer calibration methods are the socalled Langley calibrations (and its variations), which are based on extrapolating the diurnal SPM signal to zero air mass (e.g. Forgan, 1994). While no additional equipment is needed, the Langley methods require clear and highly stable atmospheric aerosol conditions, typically only found above the planetary boundary layer on high mountain sites.

Here, we introduce a new method that may alleviate this restriction and improve the SPM calibration beyond the Langley uncertainty.

\section{Systematic diurnal variations of AOD and Ångström parameters}

A ground-based photo detector pointed at the sun with a narrow field-of-view and a band pass filter measures a signal $V$ that is related to the signal at the top of the atmosphere $V_{0}$ (the calibration constant, sometimes also called extraterrestrial signal) by

$V=V_{0} / r^{2} \times \exp (-\tau m)$,

where $r$ is the normalized Sun-Earth distance and $m$ is the relative air mass factor. The air mass is approximately $1 / \cos (\theta), \theta$ is the solar zenith angle (SZA), and refined expressions are given by Kasten and Young (1989). The total extinction $\tau$ is the sum of the contributions of aerosol optical depth $\tau_{\mathrm{a}}$ and $\tau_{\text {ray }}$, the extinction due to molecular Rayleigh scattering (Bodhaine et al., 1999). Here, for simplicity and clarity of the argument, we restrict the discussion to bands outside molecular absorptions.

The logarithm of the signal $V$ has a linear diurnal dependency on the air mass with slope $\tau$ and ordinate intercept $\log \left(V_{0} / r^{2}\right)$. So the calibration constant $V_{0}$ can be found by a linear fit, i.e. extrapolating to zero air mass, which is the basis of the Langley calibration methods. Langley calibrations 
at high mountain sites (e.g. Mauna Loa, Hawaii or Jungfraujoch, Swiss Alps) are amongst the most accurate methods for SPM calibration and achieve uncertainties below $0.5 \%$ (Holben et al., 1998; Schmid et al., 1998; Wehrli, 2000).

Knowing $V_{0}$, the AOD can be calculated straightforwardly by rearranging Eq. (1):

$\tau_{\mathrm{a}}=-\log \left(r^{2} V / V_{0}\right) / m-\tau_{\text {ray }}$.

Errors in the calibration are typically the largest sources of uncertainty in determining AOD (Shaw, 1976). It follows from Eq. (2) that an error in $V_{0}$ causes an air mass dependent offset in the derived AOD, which has been pointed out by several authors (see, e.g. Cachorro et al., 2008). The erroneously derived AOD, $\tau_{\mathrm{a}}^{\prime}$ under the assumption of a wrong $V_{0}^{\prime}$ is

$\tau_{\mathrm{a}}^{\prime}=\tau_{\mathrm{a}}+\log \left(V_{0}^{\prime} / V_{0}\right) / m$.

A calibration error manifests itself in an artificial diurnal variation (DV) of the derived AOD. The deviation has a maximal magnitude at noon (bigger for higher solar elevations) and vanishes for large air masses at sunset/sunrise and is symmetrical around local solar noon. Cachorro et al. (2008) have proposed an improvement of the calibration from that effect, but like all variations of the Langley method, it is intrinsically limited by the natural variations of AOD.

Here we consider the spectral AOD relation because it is not affected by AOD variations if the aerosol type does not change. The AOD dependency on wavelength is usually described by the Ångström exponent $\alpha$ (Ångström, 1964). With Ångström's power-law ansatz, the $\log \tau_{\mathrm{a}}-\log \lambda$ relationship is linear with slope $\alpha$ :

$\log \tau_{\mathrm{a}}=\log \beta-\alpha \log \lambda$,

with $\lambda$ in units of microns, and where $\beta$ is the AOD at wavelength of one micron. It has become common (e.g. Schuster et al., 2006) to add a quadratic term in $\log \lambda$ to account for a possible curvature:

$\log \tau_{\mathrm{a}}=\log \beta^{*}+\alpha^{*} \log \lambda+\gamma \log ^{2} \lambda$.

The parameters $\alpha$ and $\gamma$ are determined by regression of Eqs. (4) and (5), respectively. To investigate the sensitivity of AOD, $\alpha$ and $\gamma$ on the calibration, we theoretically simulate the SPM measurement and retrieval process. We consider the case of the precision filter radiometer SPM (PFR-SPM) developed by the Physikalisch-Meteorologisches Observatorium (PMOD) in Davos, Switzerland, for the Global Atmospheric Watch Network (Wehrli, 2000). It features four standard aerosol wavelength channels: $368 \mathrm{~nm}, 412 \mathrm{~nm}, 501 \mathrm{~nm}$ and $862 \mathrm{~nm}$, which will be referred to as channels 1 to 4 , respectively. For comparison, commonly used wavelengths measured by the AErosol RObotic NETwork are $380 \mathrm{~nm}$, $440 \mathrm{~nm}, 500 \mathrm{~nm}, 670 \mathrm{~nm}$ and $870 \mathrm{~nm}$ (Holben et al., 1998).
We simulate the diurnal signal $V$ for all wavelengths for a given spectral $\tau_{\mathrm{a}}$ using Eq. (1) and a hypothetical wrong calibration constant $V_{0}^{\prime}$ of channel 4 at $862 \mathrm{~nm}$ of $V_{0}+\sigma$ and $V_{0}-\sigma$, where $\sigma$ is the calibration uncertainty of $0.5 \%$. From Eq. (2) we derive the erroneous spectral AOD $\tau_{\mathrm{a}}^{\prime}$ under the assumption of $V_{0}^{\prime}=V_{0}$, from which we obtain the (erroneous) $\alpha^{\prime}$ and $\gamma^{\prime}$.

Figure 1 shows the calculation for the example values: $\beta^{*}=0.02, \alpha^{*}=2$, and $\gamma=-0.5$, which constitute pristine conditions. The time (UT) corresponds to a SZA between $87^{\circ}$ and $63^{\circ}$ or air mass between 23 and 2.2 at noon (11:25 UT), beginning of November at the location of Innsbruck, Austria, at $11^{\circ} \mathrm{E}$ and $47^{\circ} \mathrm{N}$.

All parameters show the characteristic DV symmetric around noon. The deviation of AOD, $\alpha$ and $\gamma$ is biggest at local noon (DV amplitude) and approaches the correct values at low solar elevation. The respective effect of $V_{0}+\sigma$ and $V_{0}-\sigma$ traces two branches marking the bounds of possible DVs, which are statistically allowed by the calibration uncertainty.

The example reveals that the DV in $\alpha$ and, in particular, $\gamma$ can be considerable, even if $V_{0}^{\prime}$ lies within the $V_{0}$ uncertainty. The DV amplitude of $\alpha$ and $\gamma$ can reach 0.1 and 0.2, respectively. The amplitude of the DV in AOD $(\sim 0.002$ for channel 4) is negligibly small and will likely be obscured by natural variations.

This DV in $\alpha$ and $\gamma$ is also of specific relevance as these parameters are used as indicators of the size or size distribution of aerosols. King et al. (1978) have introduced a numerical inversion of the spectral AOD to obtain the aerosol size distribution. A semiquantitative graphical method has been proposed (Schuster et al., 2006, and Gobbi et al., 2007), where $\gamma$ (or equivalently, the $\alpha$-difference $\mathrm{dA}$ ) is plotted against $\alpha$. Changes in fine mode size and fraction trace curved lines of a grid. Since here we use the more universal parameter of the curvature $\gamma$ instead of $\mathrm{dA}$, we call this alpha-gamma representation AGa-plot (in allusion to the AdA-plot in Gobbi's nomenclature).

With its appealing simplicity, this representation reveals, for example, diurnal aerosol evolution such as humidification or drying. In this context, calibration errors and resulting artificial DVs of $\alpha$ and $\gamma$ are specifically relevant, because they modify the AGa-plot and its interpretation.

The individual effect of a calibration error in each of the different channels on the AGa-plot is illustrated in Fig. 2. Each colored line represents the resulting DV when adding an error of $1 \sigma(0.5 \%)$ to the $V_{0}$ of the respective channel. It is apparent that an error in channel 3 or 4 (green and red lines) has the largest impact on the DV in $\alpha$ and $\gamma$, while channel 2 has a vanishing influence. The total effect of errors in all channels and the resulting error in the AGa-plot is the sum of the individual effects. 


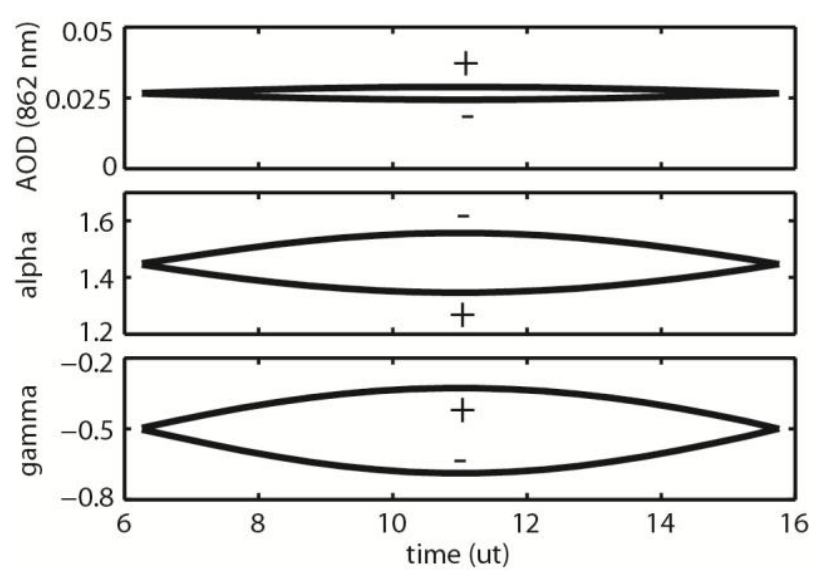

Fig. 1. Simulated DV of $\mathrm{AOD}^{\prime}, \alpha^{\prime}$ and $\gamma^{\prime}$ for a calibration error in PFR-SPM channel 4 of $V_{0}^{\prime}=V_{0}+\sigma$ and $V_{0}-\sigma$, respectively.

\section{Minimization of Diurnal Variations}

As demonstrated, a residual artificial DV in $\alpha$ and $\gamma$ may persist even after state-of-the-art calibration, showing that these parameters are extremely sensitive to calibration errors. The idea here is to harness this sensitivity and improve the calibration by minimizing any residual DV in $\alpha$ and $\gamma$. Consequently, this also improves the accuracy of the AGa representation and its interpretation. The question is, can one find a set of $V_{0}$ for all channels as close as possible to the certified calibration so that DVs in $\alpha$ and $\gamma$ both vanish?

We propose to solve the problem with a conceptually simple Monte Carlo (MC) method: trying a large ensemble of random combinations of $V_{0}^{\prime}$ weighted with a Gaussian uncertainty function centered on $V_{0}$, and select the $V_{0}^{\prime}$ that yields the smallest DV. Since both $\alpha$ and $\gamma$ show independent DVs, we consider the total DV (TDV) amplitude to be minimized: $\mathrm{TDV}^{2}=\mathrm{DV}_{\alpha}^{2}+\mathrm{DV}_{\gamma}^{2}$. The DV amplitudes $\mathrm{DV}_{\alpha}$ and $\mathrm{DV}_{\gamma}$ are specified here as their respective difference of their values at noon (lowest SZA) and highest SZA. An ensemble of diurnal $\alpha$ and $\gamma$ values from 100 generated $V_{0}^{\prime}$ is shown in Fig. 2. The DVs are arranged in a "star" pattern, with the center being the true $\alpha$ and $\gamma$ values approached at low solar elevation and the length of the spikes are the TDV amplitudes.

Note that using a random number for $V_{0}^{\prime}$ that is normally distributed with $\sigma$ is an implicit weighting of the solution by the mean squared error of $V_{0}$ and $V_{0}^{\text {best }}$. Smaller deviations of $V_{0}^{\prime}$ from $V_{0}$ are more likely generated in the MC method, and hence the solution $V_{0}^{\text {best }}$ should be found as close to $V_{0}$ as possible.

\section{Application to SPM data}

We then apply the above MC method of finding an improved $V_{0}$ with resulting minimal TDV to real data of our SPM

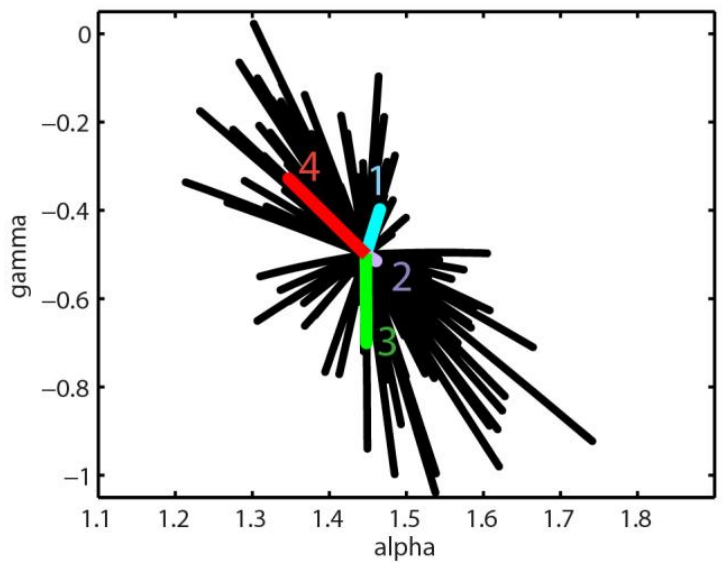

Fig. 2. Calibration error effect in the AGa representation of $V_{0}^{\prime}=$ $V_{0}+\sigma$ in each individual PFR-SPM channel (colored lines) and that of an ensemble of 100 normally distributed random $V_{0}$ errors in all channels (black lines).

located in Innsbruck, Austria. We have been measuring AOD since 2007 and the 5-yr climatology has recently been published (Wuttke et al., 2012).

The SPM has been calibrated at the PMOD by comparison with two Langley calibrated standard instruments. $V_{0}$ for the four wavelengths channels from the certificate is

$V_{0}^{C}=(4.262(20) 3.778(16) 3.772(30) 3.565(20)) V$,

where the $1 \sigma$ uncertainties are given in brackets, corresponding to relative accuracies in $V_{0}$ of $0.5 \%, 0.4 \%, 0.8 \%$ and $0.6 \%$, respectively.

We found a particularly good example day in Innsbruck with low and fairly constant AOD on 10 November 2011. This cloudless day in late autumn with relatively low solar elevation was characterized by a dominant anticyclonic weather situation, no wind and no convective mixing of the boundary layer.

We define $\mathrm{DV}_{\alpha}$ as the difference of $\alpha$ at noon (mean of $24 \mathrm{~min}$ ) and the mean of $\alpha$ at three hours before and after noon. $\mathrm{DV}_{\gamma}$ is defined accordingly and again the TDV amplitude is considered. We run the MC procedure with 10000 trials (different combinations of weighted random $V_{0}^{\prime}$ ) to select the $V_{0}^{\prime}$ with the minimal TDV amplitude and find

$V_{0}^{\text {best }}=\left(\begin{array}{llll}4.277 & 3.795 & 3.730 & 3.583\end{array}\right) V$.

The difference to the original certificate is $\left(V_{0}^{C}-V_{0}^{\text {best }}\right)=$ $\left(\begin{array}{llll}-0.015 & -0.017 & 0.043 & -0.018\end{array}\right) V$, which lies almost within the $1 \sigma$ uncertainty of the calibration certificate. The effect of applying this new $V_{0}^{\text {best }}$ to the derivation of the aerosol parameters is shown in Fig. 3a (green curves). The absolute difference in resulting AOD is (0.002 0.0020 .005 0.002). These differences are of the order of the given AOD uncertainty and smaller than the standard deviation of the 

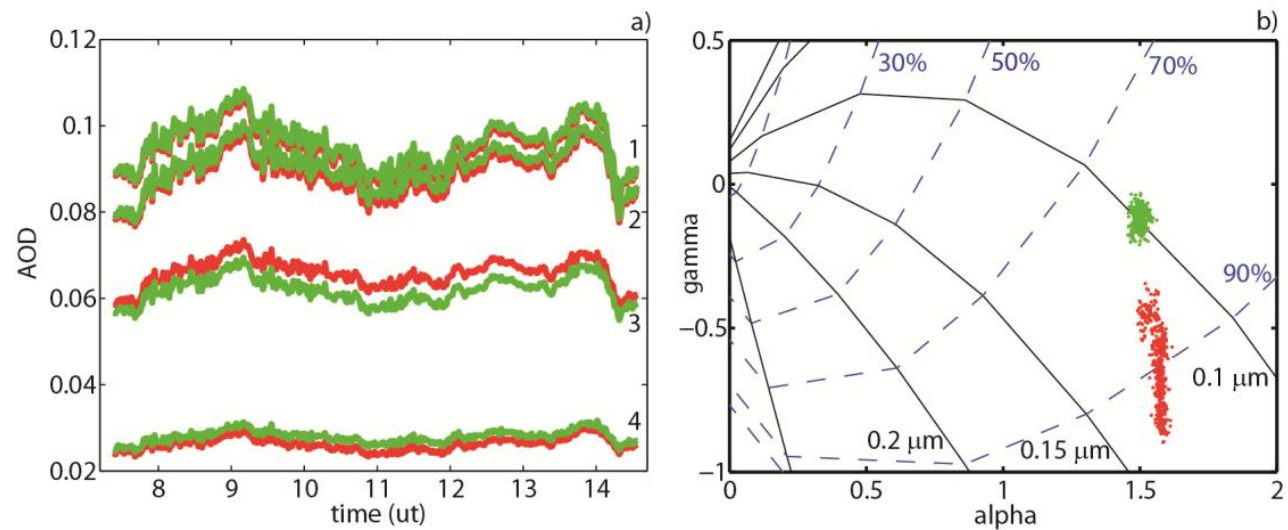

Fig. 3. (a) SPM data of 10 November 2011 used for improving the calibration by minimizing the TDV amplitude with a MC method. The difference of retrieved AOD with $V_{0}^{C}$ (red) and the new $V_{0}^{\text {best }}$ (green) is within the measurement uncertainty. (b) The effect of the improved calibration on the AGa-plot is significant. Notably, the green points are more clustered and shifted to a larger $\gamma$ value. The grid lines refer to fine mode radius $(\mu \mathrm{m})$ and fraction $(\%)$.

AODs (variation) over the day of each channel (except for channel 3 where the standard deviation of AOD is 0.03). No significant DV of AOD can be detected within the natural fluctuations.

The data for $\alpha$ and $\gamma$ are depicted in the AGa-plot in Fig. 3b. The original DV (red dots) in $\gamma$ is significant, as it ranges from -0.4 in the morning to -0.9 at noon. The variation in $\alpha$ is less and ranges from 1.5 to 1.6. Consequently, in the AGa-plot the representation has changed significantly after application of $V_{0}^{\text {best }}$. The loci of green points are now more clustered (standard deviations of $\alpha$ and $\gamma$ are 0.02 and 0.03 , respectively) and indicate a larger $\gamma$, pointing towards a smaller fine mode radius and fraction.

\section{Discussion}

One of the questions after finding a solution with minimal TDV and corresponding $V_{0}^{\text {best }}$ is about its uniqueness. One might conclude that a $V_{0}^{\prime}$ that minimizes the TDV is necessarily closer to the true $V_{0}$. This seems not true in general.

The calibration error in each channel results in a different $\mathrm{TDV}$, both in magnitude and direction in the AGa representation (Fig. 2). This means that a given TDV can be realized by a variety of combinations in $V_{0}^{\prime}$, and hence, the sole criterion of a minimal TDV does not yield a unique solution $V_{0}^{\text {best }}$. It is only the result of the additional constraint of minimizing the root-mean-squared error (rms) with respect to the original $V_{0}$ (realized by the normal distribution of trials in the MC method) that a unique solution can be found. Of course, for a $V_{0}^{\text {best }}$ with a large rms distance to the true calibration, the DV in the AOD will become significant and can then be used as a constraint.

The next question is about the uncertainty of the solution $V_{0}^{\text {best }}$ and whether it really constitutes an improvement beyond the Langley uncertainty. We repeat the MC procedure with 100 trials of simulated data for 100 random $V_{0}$ in the interval $\left(V_{0}-\sigma, V_{0}+\sigma\right)$ and compare each solution $V_{0}^{\text {best }}$ with the corresponding target input $V_{0}$. The standard deviation of the difference of the two is the uncertainty of $V_{0}^{\text {best }}$. We find that channels 1 and 2 have similar standard deviations as the $\sigma$ of $0.5 \%$. The standard deviation of channels 3 and 4 are determined to $0.31 \%$ and $0.14 \%$, respectively. This implies that the $V_{0}^{\text {best }}$ of channels 3 and 4 are indeed closer to the true $V_{0}$ and reduce their calibration uncertainty to $0.61 \sigma$ and $0.28 \sigma$, respectively.

The reason why the channels at higher wavelengths are more sensitive here, is that their wavelength spacing (in logarithmic scale) increases with wavelength. The logarithmic distance between channels 1 and 2 is $1 / 5$ of that of channel 3 and 4 . The latter are hence more influential on the determination of $\alpha$ and $\gamma$, and their calibration is more sensitive.

The reduction of the calibration uncertainty achieved here stems from the consideration of the spectral AOD, i.e. the combination of all channels as opposed to each channel individually in the Langley methods. The spectral AOD relations are more sensitive to calibration errors and independent of natural AOD variations. Langley conditions (constant AOD) are hardly met at low elevation stations, while constant spectral AOD conditions do occur more frequently. This is an empirical observation based on our aerosol climatology in Innsbruck, and we do not see any principal reason why it should not be more generally applicable to other stations. On suitable days for this method, such as our example day, the residual variation in $\alpha$ and $\gamma$ should be small after minimizing the TDV (green points in Fig. 3b).

Finally, we note that our method does not in principle rely on a precalibrated instrument. Without any a priori knowledge of $V_{0}^{C}$, however, the number of required MC trials may be too large to be tractable for computation. A deterministic optimization procedure would be better suited in such a case. 


\section{Conclusions}

We have shown that the Ångström parameters $\alpha$ and $\gamma$ are extremely sensitive indicators of calibration errors and artificial DVs can be significant under low AOD conditions, even if the calibration is within $0.5 \%$ relative uncertainty. The DV amplitudes in $\alpha$ and $\gamma$ can be reduced by stochastically varying the calibration constant $V_{0}$ using a weighted MC method. The method has been characterized with model simulations and applied to real data from a PFR-SPM on a clear and stable day in Innsbruck. Minimizing the total DV amplitude in $\alpha$ and $\gamma$, a new calibration constant $V_{0}$ has been determined which reduces the calibration uncertainty of two of the PFR-SPM wavelengths channels by a factor of up to 3.6. Our method may be easily generalized to other SPMs with more and/or different aerosol wavelength channels to improve the calibration beyond the Langley uncertainty.

Acknowledgements. We thank G.P. Gobbi for the calculation of the grid lines in the AGa-plots.

Edited by: S. Schmidt

\section{References}

Ångström, A.: The parameters of atmospheric turbidity, Tellus, 16, 64-75, 1964.

Bodhaine, B. A., Wood, N. B., Dutton, E. G., and Slusser, J. R.: On Rayleigh Optical Depth Calculations, J. Atmos. Ocean. Tech. 16, 1854-1861, 1999.

Cachorro, V. E., Toledano, C., Berjon, A., de Frutos, A. M., Torres, B., Sorribas, M., and Laulainen, N. S.: An "in situ" calibrationcorrection procedure (KCICLO) based on AOD diurnal cycle: Comparative results between AERONET and reprocessed (KCICLO method) AOD-alpha data series at El Arenosillo, Spain, J. Geophys. Res. 113, D02207, doi:10.1029/2007JD009001, 2008.
Forgan, B. W.: General method for calibrating Sun photometers, Appl. Optics, 33, 4841-4850, 1994.

Forster, P., Ramaswamy, V., and Artaxo, P., et al.: Changes in Atmospheric Constituents and in Radiative Forcing, in: Climate Change 2007: The Physical Science Basis, Contribution of WG I to the Fourth AR of the IPCC, Cambridge University Press, Cambridge, United Kingdom and New York, NY, USA, 2007.

Gobbi, G. P., Kaufman, Y. J., Koren, I., and Eck, T. F.: Classification of aerosol properties derived from AERONET direct sun data, Atmos. Chem. Phys., 7, 453-458, doi:10.5194/acp-7-453-2007, 2007.

Holben, B. N., Eck, T.F., Slutsker, I., Tanre, D., Buis, J. P., Setzer, A., Vermote, E., Reagan, J. A., Kaufman, Y. J., Nakajima, T., Lavenu, F., Jankowiak, I., and Smirnov, A.: AERONET- A federated instrument network and data archive for aerosol characterization, Remote Sens. Env., 66, 1-16, doi:10.1016/S00344257(98)00031-5, 1998.

Kasten, F. and Young, A. T.: Revised optical air mass tables and approximation formula, Appl. Optics, 28, 4735-4738, 1989.

King, M. D., Byrne, D. M., Herman, B. M., and Reagan, J. A.: Aerosol Size Distributions Obtained by Inversion of Spectra Optical Depth Measurements, J. Atmos. Sci. 35, 2153-2167, 1978.

Schmid, B., Spyak, P. R., Biggar, S. F., Wehrli, C., Sekler, J., Ingold, T., Mätzler, C., and Kämpfer, N.: Evaluation of the applicability of solar and lamp radiometric calibrations of a precision Sun photometer operating between 300 and $1025 \mathrm{~nm}$, Appl. Optics, 37, 3923-3941, 1998

Schuster, G. L., Dubovik, O., and Holben, B. N.: Ångström exponent and bimodal aerosol size distributions, J. Geophys. Res., 111, 1-14, doi:10.1029/2005JD006328, 2006.

Shaw, G. E.: Error analysis of multi-wavelength sun photometry, Pure Appl. Geophys., 114, 1-14, 1976.

Wehrli, C.: Calibrations of filter radiometers for determination of atmospheric optical depth, Metrologia, 37, 419-422, 2000.

Wuttke, S., Kreuter, A., and Blumthaler, M.: Aerosol climatology in an alpine valley, J. Geophys. Res., 117, D20202, doi:10.1029/2012JD017854, 2012. 